PROCEEDINGS OF THE

AMERICAN MATHEMATICAL SOCIETY

Volume 137, Number 3, March 2009, Pages 1093-1102

S 0002-9939(08)09680-9

Article electronically published on October 21, 2008

\title{
EIGENVALUE ESTIMATES FOR SUBMANIFOLDS WITH LOCALLY BOUNDED MEAN CURVATURE IN $N \times \mathbb{R}$
}

\author{
G. PACELLI BESSA AND M. SILVANA COSTA
}

(Communicated by Richard A. Wentworth)

\begin{abstract}
We give lower bounds for the fundamental tone of open sets in submanifolds with locally bounded mean curvature in $N \times \mathbb{R}$, where $N$ is an $n$-dimensional complete Riemannian manifold with radial sectional curvature $K_{N} \leq \kappa$. When the immersion is minimal our estimates are sharp. We also show that cylindrically bounded minimal surfaces have a positive fundamental tone.
\end{abstract}

\section{INTRODUCTION}

The fundamental tone $\lambda^{*}(\Omega)$ of an open set $\Omega$ in a smooth Riemannian manifold $M$ is defined by

$$
\lambda^{*}(\Omega)=\inf \left\{\frac{\int_{\Omega}|\operatorname{grad} f|^{2}}{\int_{\Omega} f^{2}} ; f \in H_{0}^{1}(\Omega) \backslash\{0\}\right\} .
$$

When $\Omega=M$ is an open Riemannian manifold, the fundamental tone $\lambda^{*}(M)$ coincides with the greatest lower bound inf $\Sigma$ of the spectrum $\Sigma \subset[0, \infty)$ of the unique self-adjoint extension of the Laplacian $\triangle$ acting on $C_{0}^{\infty}(M)$, also denoted by $\triangle$. When $\Omega$ is compact with piecewise smooth boundary $\partial \Omega$ (possibly empty), $\lambda^{*}(\Omega)$ is the first eigenvalue $\lambda_{1}(\Omega)$ of $\Omega$ (Dirichlet boundary data if $\partial \Omega \neq \emptyset$ ). A well studied problem in the geometry of the Laplacian is the relation between the first eigenvalue/fundamental tone of open sets of a Riemannian manifold and its geometric invariants; see [2], 3], 8] and the references therein. Another interesting problem is to give bounds for the the first eigenvalue/fundamental tone of open sets of minimal submanifolds of Riemannian manifolds; see [4, [5, [7, 9], 10]. There has recently been increasing interest in the study of minimal surfaces (constant mean curvature) in product spaces $N \times \mathbb{R}$, after the discovery of many beautiful examples in those spaces; see [16, [17. This motivates us to study the fundamental tone of minimal submanifolds of product spaces $N \times \mathbb{R}$. We prove the following theorem.

Theorem 1.1. Let $\varphi: M \hookrightarrow N \times \mathbb{R}$ be a complete minimal m-dimensional submanifold, where $N$ has radial sectional curvature $K(\gamma(t))\left(\gamma^{\prime}(t), v\right) \leq \kappa, v \in T_{\gamma(t)} N$,

Received by the editors April 29, 2008.

2000 Mathematics Subject Classification. Primary 53C40, 53C42; Secondary 58C40.

Key words and phrases. Fundamental tone estimates, minimal submanifolds, submanifolds with locally bounded mean curvature in $N \times \mathbb{R}$.

The first author was partially supported by a CNPq-grant and ICTP Associate Schemes.

The second author was partially supported by a CNPq-scholarship. 
$|v|=1, v \perp \partial t$, along the geodesics $\gamma(t)$ issuing from a point $x_{0} \in N$. Let $\Omega \subset \varphi^{-1}\left(B_{N}\left(x_{0}, r\right) \times \mathbb{R}\right)$ be a connected component, where $r<\min \left\{\operatorname{inj}\left(x_{0}\right), \pi / 2 \sqrt{\kappa}\right\}$ $(\pi / 2 \sqrt{\kappa}=\infty$ if $\kappa \leq 0)$. Then

$$
\lambda^{*}(\Omega) \geq \lambda_{1}\left(B_{\mathbb{N}^{m-1}(\kappa)}(r)\right) .
$$

If $\Omega$ is bounded, then inequality (1.1) is strict. Here $\mathbb{N}^{m-1}(\kappa)$ is the $(m-1)$ dimensional simply connected space form of constant sectional curvature $\kappa$.

Theorem 1.1 can be viewed as a version of Theorem 1.10 of [5] for product spaces. There Bessa and Montenegro gave eigenvalue estimates for pre-images of geodesic balls in Riemannian manifolds with radial sectional curvature bounded above. Here we give lower eigenvalue estimates for pre-images of cylinders in product spaces.

Remark 1.2. Inequality (1.1) is sharp. For if we let $\varphi: \mathbb{H}^{m-1}(-1) \times \mathbb{R} \hookrightarrow \mathbb{H}^{n}(-1) \times \mathbb{R}$ be given by $\varphi(x, t)=(i(x), t)$, where $i: \mathbb{H}^{m-1}(-1) \hookrightarrow \mathbb{H}^{n}(-1)$ is a totally geodesic embedding, then for $\Omega=\varphi^{-1}\left(B_{\mathbb{H}^{n}(-1)}(r) \times \mathbb{R}\right)=B_{\mathbb{H}^{m-1}(-1)}(r) \times \mathbb{R}$ we have

$$
\lambda^{*}(\Omega)=\lambda_{1}\left(B_{\mathbb{H}(-1)^{m-1}}(r)\right) .
$$

Corollary 1.3. Let $\varphi: M \hookrightarrow \mathbb{R}^{3}$ be a complete minimal surface with $\varphi(M) \subset$ $B_{\mathbb{R}^{2}}(r) \times \mathbb{R}$. Then

$$
\lambda^{*}(M) \geq \lambda_{1}\left(B_{\mathbb{R}^{2}}(r)\right)=\frac{c_{0}}{r^{2}},
$$

where $c_{0}$ is the first zero of the $J_{0}$-Bessel function.

Question 1.4. The only examples of complete surfaces in $\mathbb{R}^{3}$ with positive fundamental tone (to the best of our knowledge) are the Nadirashvili bounded minimal surfaces [18, the Martin-Morales cylindrically bounded minimal surfaces [15] and the unearthly examples of Lopez-Martin-Morales bounded minimal surfaces, 13], 14, where they added handles to Nadirashvillli bounded minimal surfaces and constructed complete nonorientable bounded minimal surfaces in $\mathbb{R}^{3}$. These minimal surfaces have at least two bounded coordinate functions, and that is crucial in the proof that their fundamental tones are positive. This raises the question as to whether there are minimal surfaces in $\mathbb{R}^{3}$ with at most one bounded coordinate function with positive fundamental tone. In particular one can ask: has the Jorge-Xavier minimal surface inside the slab 12 positive fundamental tone?

A second purpose of this paper is study the fundamental tones of domains in submanifolds with locally bounded mean curvature in $N \times \mathbb{R}$. This is a stronger notion than the locally bounded mean curvature considered in [4].

Definition 1.5. An immersed submanifold $\varphi: M \hookrightarrow N \times \mathbb{R}$ has locally bounded mean curvature $|H|$ in $N \times \mathbb{R}$ if for any $p \in N$ and $r>0$, the number

$$
h(p, r)=\sup \left\{|H(x)| ; x \in \varphi(M) \cap\left(B_{N}(p, r) \times \mathbb{R}\right)\right\}
$$

is finite. Here $B_{N}\left(x_{0}, r\right)$ is the geodesic ball of radius $r$ and center $x_{0}$ in $N$.

Our second result is the following theorem.

Theorem 1.6. Let $\varphi: M \hookrightarrow N \times \mathbb{R}$ be a complete immersed $m$-submanifold with locally bounded mean curvature in $N \times \mathbb{R}$, where $N$ has radial sectional curvature $K \leq$ $\kappa$, along the geodesics issuing from a point $x_{0} \in N$. Let $\Omega(r) \subset \varphi^{-1}\left(B_{N}(p, r) \times \mathbb{R}\right)$ be any connected component with $r \leq \min \left\{\operatorname{inj}_{N}\left(x_{0}\right), \pi / 2 \sqrt{\kappa}\right\}$. Suppose in addition that 
- if $\left|h\left(x_{0}, r\right)\right|<\Lambda^{2}<\infty$, then $r \leq\left(C_{\kappa} / S_{\kappa}\right)^{-1}\left(\Lambda^{2} /(m-2)\right)$ or

- if $\lim _{r \rightarrow \infty} h\left(x_{0}, r\right)=\infty$, then $r \leq\left(C_{\kappa} / S_{\kappa}\right)^{-1}\left(h\left(x_{0}, r_{0}\right) /(m-2)\right)$, where $r_{0}$ is so that $(m-2) \frac{C_{\kappa}}{S_{\kappa}}\left(r_{0}\right)-h\left(x_{0}, r_{0}\right)=0$.

Then we have

$$
\lambda^{*}(\Omega(r)) \geq\left[\frac{(m-2) \frac{C_{\kappa}}{S_{\kappa}}(r)-h\left(x_{0}, r\right)}{2}\right]^{2}>0 .
$$

Corollary 1.7 (Bessa-Montenegro, [6]). Let $\varphi: M \hookrightarrow N \times \mathbb{R}$ be a compact immersed submanifold with mean curvature vector $H$. Let $p_{1}: N \times \mathbb{R} \rightarrow N$ be the projection on the first factor. Then the extrinsic radius of $p_{1}(M)$ is given as

$$
R_{p_{1}(M)}=\left(\frac{C_{\kappa}}{S_{\kappa}}\right)^{-1}\left(\sup _{M}|H| /(m-2)\right) .
$$

\section{Preliminaries}

Let $\varphi: M \hookrightarrow W$ be an isometric immersion, where $M$ and $W$ are complete Riemannian manifolds of dimension $m$ and $n$ respectively. Consider a smooth function $g: W \rightarrow \mathbb{R}$ and the composition $f=g \circ \varphi: M \rightarrow \mathbb{R}$. Identifying $X$ with $d \varphi(X)$ we have at $q \in M$ that the Hessian of $f$ is given by

$$
\text { Hess } f(q)(X, Y)=\operatorname{Hess} g(\varphi(q))(X, Y)+\langle\operatorname{grad} g, \alpha(X, Y)\rangle_{\varphi(q)} .
$$

Taking the trace in (2.1), with respect to an orthonormal basis $\left\{e_{1}, \ldots e_{m}\right\}$ for $T_{q} M$, we have the Laplacian of $f$,

$$
\Delta f(q)=\sum_{i=1}^{m} \operatorname{Hess} g(\varphi(q))\left(e_{i}, e_{i}\right)+\left\langle\operatorname{grad} g, \sum_{i=1}^{m} \alpha\left(e_{i}, e_{i}\right)\right\rangle .
$$

The formulas (2.1) and (2.2) are well known in the literature; see [11].

For the proof of Theorems 1.1 and 1.6 we will need a few preliminary results. The first result we need is the Hessian Comparison Theorem; one can see [19] for a proof.

Theorem 2.1 (Hessian Comparison Theorem). Let $W$ be a complete Riemannian manifold and let $\rho$ be the distance function on $W$ to $x_{0}$. Let $\gamma$ be a minimizing geodesic starting at $x_{0}$ and suppose that the radial sectional curvatures of $M$ along $\gamma$ are bounded above $K_{\gamma} \leq \kappa$. Then the Hessian of $\rho$ at $\gamma(t)$ satisfies

$$
\operatorname{Hess} \rho(\gamma(t))(X, X) \geq \frac{C_{\kappa}}{S_{\kappa}}(t) \cdot\|X\|^{2}, \quad X \perp \gamma^{\prime}(t),
$$

where

$$
S_{\kappa}(t)= \begin{cases}\sin (\sqrt{\kappa} \cdot t) / \sqrt{\kappa} & \text { if } \quad \kappa>0 \\ 1 / t & \text { if } \kappa=0 \\ \sinh (\sqrt{-\kappa} \cdot t) / \sqrt{-\kappa} & \text { if } \quad \kappa<0\end{cases}
$$

and $C_{\kappa}(t)=S_{\kappa}^{\prime}(t)$ 
The second and third results we need are eigenvalue estimates proved in [5] and in [4. The former is a generalization of the well known Barta's eigenvalue theorem [1, and the later is a generalization of a result of Cheung-Leung [10].

Theorem 2.2 ([5]). Let $\Omega$ be an open set in a Riemannian manifold $M$. Then

$$
\lambda^{*}(\Omega) \geq \sup _{\mathcal{C}(\Omega)}\left\{\inf _{\Omega}\left(\operatorname{div} X-|X|^{2}\right)\right\}
$$

where $\mathcal{C}(\Omega)$ is the set of smooth vector fields in $\Omega \backslash F$ for some closed set $F$ with Hausdorff measure $\mathcal{H}^{n-1}(F \cap \Omega)=0$.

Theorem 2.3 (4]). Let $\Omega$ be an open set in a Riemannian manifold $M$ and $c(\Omega)$ a constant defined by

$$
c(\Omega)=\sup _{\mathcal{C}_{+}(\Omega)} \frac{\left(\inf _{\Omega} \operatorname{div} X\right)}{\sup _{\Omega}|X|^{2}},
$$

where $\mathcal{C}_{+}(\Omega)=\left\{X \in \mathcal{C}(\Omega) \inf _{\Omega} \operatorname{div} X>0\right.$ and $\left.\sup _{\Omega}|X|<\infty\right\}$. Then

$$
\lambda^{*}(\Omega) \geq \frac{c(\Omega)^{2}}{4} .
$$

Finally, the fourth result we need is the following technical lemma.

Lemma 2.4. Let $v: B_{\mathbb{N}^{n}(\kappa)}(r) \rightarrow \mathbb{R}$ be a first positive eigenfunction of $B_{\mathbb{N}^{n}(\kappa)}(r) \subset$ $\mathbb{N}^{n}(\kappa)$ associated to the first eigenvalue $\lambda_{1}\left(B_{\mathbb{N}^{n}(\kappa)}(r)\right)$. Then

$$
n \frac{C_{\kappa(t)}}{S_{\kappa}(t)} v^{\prime}(t)+\lambda_{1}\left(B_{\mathbb{N}^{n}(\kappa)}(r)\right) v(t)<0, \quad t \in(0, r) .
$$

Proof. We are going to treat the cases $\kappa<0, \kappa=0$ and $\kappa>0$ separately. Suppose first that $\kappa<0$ and let us call $\lambda=\lambda_{1}\left(B_{\mathbb{N}^{n}(\kappa)}(r)\right)$ for simplicity of notation. Recall that $v(t)$ satisfies the following differential equation:

$$
v^{\prime \prime}(t)+(n-1) \frac{C_{\kappa}}{S_{\kappa}}(t) v^{\prime}(t)+\lambda v(t)=0, \quad t \in(0, r) .
$$

Consider the function $\mu(t)=C_{\kappa}(t)^{\frac{\lambda}{n \kappa}}$. Thus $\mu^{\prime}(t)=-\frac{\lambda}{n} S_{\kappa}(t) C_{\kappa}(t)^{\frac{\lambda}{n \kappa}-1}$ and

$$
\begin{aligned}
v^{\prime}(t) \mu(t)-\mu^{\prime}(t) v(t) & =v^{\prime}(t) C_{\kappa}(t)^{\frac{\lambda}{n \kappa}}+\frac{\lambda}{n} S_{\kappa}(t) C_{\kappa}(t)^{\frac{\lambda}{n \kappa}-1} v(t) \\
& =\frac{1}{n} C_{\kappa}(t)^{\frac{\lambda}{n \kappa}-1} S_{\kappa}(t)\left(n \frac{C_{\kappa}(t)}{S_{\kappa}(t)} v^{\prime}(t)+\lambda v(t)\right) .
\end{aligned}
$$

From (2.9) we see that to prove that $n \frac{C_{\kappa}(t)}{S_{\kappa}(t)} v^{\prime}(t)+\lambda v(t)<0$ we only need to prove

$$
v^{\prime}(t) \mu(t)-\mu^{\prime}(t) v(t)<0
$$

Multiplying equation (2.8) by $S_{\kappa}^{n-1}$, we obtain the following differential equation:

$$
\left(S_{\kappa}^{n-1} v^{\prime}\right)^{\prime}(t)+\lambda S_{\kappa}^{n-1}(t) v(t)=0, \quad t \in(0, r) .
$$

The function $\mu(t)=C_{\kappa}(t)^{\frac{\lambda}{n \kappa}}$ satisfies the differential equation

$$
\mu^{\prime \prime}(t)=-\lambda\left(\frac{1}{n C_{\kappa}^{2}(t)}-\frac{\lambda}{n^{2}} \frac{S_{\kappa}^{2}(t)}{C_{\kappa}^{2}(t)}\right) \mu(t) .
$$

Multiplying equation (2.11) by $S_{\kappa}^{n-1}(t)$ we obtain

$$
S_{\kappa}^{n-1}(t) \mu^{\prime \prime}(t)+\lambda S_{\kappa}^{n-1}(t)\left(\frac{1}{n C_{\kappa}^{2}(t)}-\frac{\lambda}{n^{2}} \frac{S_{\kappa}^{2}(t)}{C_{\kappa}^{2}(t)}\right) \mu(t)=0 .
$$


Adding and subtracting the term $(n-1) \mu^{\prime}(t) S_{\kappa}^{n-2}(t) C_{\kappa}(t)$ we obtain

$$
\left(S_{\kappa}^{n-1} \mu^{\prime}\right)^{\prime}(t)+\lambda S_{\kappa}^{n-1}(t)\left(\frac{n-1}{n}+\frac{1}{n C_{\kappa}^{2}(t)}-\frac{\lambda}{n^{2}} \frac{S_{\kappa}^{2}(t)}{C_{\kappa}^{2}(t)}\right) \mu(t)=0 .
$$

The functions $v$ and $\mu$ then satisfy the follow identities:

$$
\begin{aligned}
\left(S_{\kappa}^{n-1} v^{\prime}\right)^{\prime}(t)+\lambda S_{\kappa}^{n-1}(t) v(t) & =0, \\
\left(S_{\kappa}^{n-1} \mu^{\prime}\right)^{\prime}(t)+\lambda S_{\kappa}^{n-1}(t)\left(\frac{n-1}{n}+\frac{1}{n C_{\kappa}^{2}(t)}-\frac{\lambda}{n^{2}} \frac{S_{\kappa}^{2}(t)}{C_{\kappa}^{2}(t)}\right) \mu(t) & =0 .
\end{aligned}
$$

Multiply the first identity of (2.14) by $\mu(t)$ and the second identity by $-v(t)$. Adding them and integrating from 0 to $t$ we obtain

$$
S_{\kappa}^{n-1}\left(v^{\prime} \mu-\mu^{\prime} v\right)(t)=-\int_{0}^{t} \lambda S_{\kappa}^{n-1}(t)\left(\frac{1}{n}-\frac{1}{n C_{\kappa}^{2}(t)}+\frac{\lambda}{n^{2}} \frac{S_{\kappa}^{2}(t)}{C_{\kappa}^{2}(t)}\right) \mu(t) v(t) d t .
$$

Clearly

$$
\lambda S_{\kappa}^{n-1}(t)\left(\frac{1}{n}-\frac{1}{n C_{\kappa}^{2}(t)}+\frac{\lambda}{n^{2}} \frac{S_{\kappa}^{2}(t)}{C_{\kappa}^{2}(t)}\right) \mu(t) v(t)>0 .
$$

Therefore we have $v^{\prime}(t) \mu(t)-\mu^{\prime}(t) v(t)<0$ for $t \in(0, r)$. This settles the case $\kappa<0$.

Suppose that $\kappa>0$. We have $S_{\kappa}(t)=\frac{1}{\sqrt{\kappa}} \sin \sqrt{\kappa} t$ for $t \in(0, r)$ with $r<\frac{\pi}{\sqrt{\kappa}}$. Define $\mu(t)=C_{\kappa}(t)^{\frac{-\lambda}{n \kappa}}$. Thus $\mu^{\prime}(t)=\frac{\lambda}{n} S_{\kappa}(t) C_{\kappa}(t)^{\frac{-\lambda}{n \kappa}-1}$. With a similar procedure we obtain that $v$ and $\mu$ satisfy the following differential identities:

$$
\begin{aligned}
\left(S_{\kappa}^{n-1} v^{\prime}\right)^{\prime}(t)+\lambda S_{\kappa}^{n-1}(t) v(t) & =0, \\
\left(S_{\kappa}^{n-1} \mu^{\prime}\right)^{\prime}(t)-\lambda S_{\kappa}^{n-1}(t)\left(\frac{n-1}{n}+\frac{1}{n C_{\kappa}^{2}(t)}+\frac{\lambda}{n^{2}} \frac{S_{\kappa}^{2}(t)}{C_{\kappa}^{2}(t)}\right) \mu(t) & =0 .
\end{aligned}
$$

In (28) we multiply the first identity by $\mu$ and the second by $-v$. Adding them and integrating from 0 to $t$ the resulting identity we obtain

$$
S_{\kappa}^{n-1}\left(v^{\prime} \mu-\mu^{\prime}(t) v\right)(t)=-\int_{0}^{t} \lambda_{1} S_{\kappa}^{n-1}(t)\left(2-\frac{1}{n}+\frac{1}{n C_{\kappa}^{2}(t)}+\frac{\lambda}{n^{2}} \frac{S_{\kappa}^{2}(t)}{C_{\kappa}^{2}(t)}\right) \mu(t) v(t) d t .
$$

The term $\lambda S_{\kappa}^{n-1}(t)\left(2-\frac{1}{n}+\frac{1}{n C_{\kappa}^{2}(t)}+\frac{\lambda}{n^{2}} \frac{S_{\kappa}^{2}(t)}{C_{\kappa}^{2}(t)}\right) \mu(t) v(t)>0$ is positive for $t \in$ $(0, r), r<\pi / 2 \sqrt{\kappa}$. Therefore we have that $v^{\prime}(t) \mu(t)-\mu^{\prime}(t) v(t)<0$ for $t \in(0, r)$, $r<\pi / 2 \sqrt{\kappa}$.

For the case $\kappa=0$ we proceed similarly. Define $\mu(t)=e^{-\frac{\lambda t^{2}}{2 n}}$. The functions $v$ and $\mu$ satisfy the following identities:

$$
\begin{aligned}
& \left(t^{n-1} v^{\prime}(t)\right)^{\prime}+\lambda t^{n-1} v(t)=0, \\
& \left(t^{n-1} \mu^{\prime}(t)\right)^{\prime}+\lambda t^{n-1}\left(1-\frac{\lambda t^{2}}{n^{2}}\right) \mu(t)=0 .
\end{aligned}
$$


In (2.18) we multiply the first identity by $\mu$ and the second by $-v$. Adding them and integrating from 0 to $t$ the resulting identity we obtain

$$
t^{n-1}\left(v^{\prime}(t) \mu(t)-v(t) \mu^{\prime}(t)\right)=-\frac{\lambda^{2}}{n^{2}} \int_{0}^{t} \mu(t) v(t)<0, \quad \forall t \in(0, r) .
$$

Then $\mu(t) v^{\prime}(t)-\mu^{\prime}(t) v(t)<0$. This proves the lemma.

\section{Proof of the RESUlts}

\subsection{Proof of Theorem 1.1.}

Proof. Let $\varphi: M \hookrightarrow N \times \mathbb{R}$ be a minimal immersion of an $m$-dimensional Riemannian manifold $M$, where $N$ is a complete Riemannian $n$-manifold with radial sectional curvature along the geodesics $\gamma(t)$ issuing from a point $x_{0} \in N$ bounded from above $K\left(\gamma^{\prime}(t), v\right) \leq \kappa, v \in T_{\gamma(t)} N,|v|=1, v \perp \partial t$. Let $\Omega \subset$ $\varphi^{-1}\left(B_{N}\left(x_{0}, r\right) \times \mathbb{R}\right), r<\min \left\{\operatorname{inj}\left(x_{0}\right), \pi / 2 \sqrt{\kappa}\right\}(\pi / 2 \sqrt{\kappa}=\infty$ if $\kappa \leq 0)$ be a connected component. Let $\rho_{N}(x)=\operatorname{dist}_{N}\left(x_{0}, x\right)$ be the distance function in $N$ to $x_{0}$ and let $v: B_{\mathbb{N}^{m-1}(\kappa)}(r) \rightarrow \mathbb{R}$ be a first positive eigenfunction associated with the first eigenvalue $\lambda_{1}\left(B_{\mathbb{N}^{m-1}(\kappa)}(r)\right)$ of the geodesic ball of radius $r$ in the simply connected $(m-1)$-dimensional space form $\mathbb{N}^{m-1}(\kappa)$ of constant sectional curvature $\kappa$. The eigenfunction $v$ is radial, i.e. $v(x)=v(|x|)$, and we can look at $v$ as it was defined in $[0, r]$ satisfying the equation

$$
v^{\prime \prime}(t)+(m-2) \frac{C_{\kappa}}{S_{\kappa}}(t) v^{\prime}(t)+\lambda_{1}\left(B_{\mathbb{N}^{m-1}(\kappa)}(r)\right) v(t)=0, \quad t \in(0, r) .
$$

Choose the first eigenfunction that satisfies the initial conditions $v(0)=1$ and $v^{\prime}(0)=0$. Define $g: B_{N}(r) \times \mathbb{R} \rightarrow \mathbb{R}$ by $g=v \circ \rho_{N} \circ p$ and $f: \Omega \rightarrow \mathbb{R}$ by $f=g \circ \varphi$, where $p: N \times \mathbb{R} \rightarrow N$ is the projection in the first factor. Setting $X=\operatorname{grad} \log f$ we have that $\operatorname{div} X-|X|^{2}=\triangle f / f$. Thus by Theorem 2.2 we have that

$$
\lambda^{*}(\Omega) \geq \inf _{\Omega}\left(-\frac{\triangle f}{f}\right) .
$$

We are going to give the lower bound $-\triangle f / f$. Let $x \in \Omega$ and $\left\{e_{1}, \ldots, e_{m}\right\}$ be any orthonormal basis for $T_{x} \Omega$. The Laplacian of $f$ at $x$ is given by

$$
\triangle_{M} f(x)=\sum_{i=1}^{m} \operatorname{Hess}_{(N \times \mathbb{R})} g(\varphi(x))\left(e_{i}, e_{i}\right)=\sum_{i=1}^{m} \operatorname{Hess}_{N} v \circ \rho_{N}(q)\left(e_{i}, e_{i}\right) .
$$

Consider the orthonormal basis $\left\{\operatorname{grad} \rho_{N}, \partial / \partial \theta_{1}, \ldots, \partial / \partial \theta_{n-1}, \partial / \partial s\right\}$ for $T_{(q, s)}(N \times \mathbb{R})$, where $\left\{\operatorname{grad} \rho_{N}, \partial / \partial \theta_{1}, \ldots, \partial / \partial \theta_{n-1}\right\}$ is an orthonormal basis for $T_{q} N$ (polar coordinates). Let $\left\{e_{1}, \ldots, e_{m}\right\}$ be an orthonormal basis for $T_{x} \Omega$ and write

$$
e_{i}=a_{i} \cdot \operatorname{grad} \rho_{N}+b_{i} \cdot \partial / \partial s+\sum_{j=1}^{n-1} c_{i}^{j} \cdot \partial / \partial \theta_{j}
$$


where $a_{i}, b_{i}, c_{i}^{j}$ are constants satisfying $a_{i}^{2}+b_{i}^{2}+\sum_{j=1}^{n-1}\left(c_{i}^{j}\right)^{2}=1, i=1, \ldots, m$. Computing $\triangle_{M} f(x)$ we have (recall that $\varphi(x)=(q, s)$, and we let $t=\rho_{N}(q)$ )

$$
\begin{aligned}
\triangle f(x) & =\sum_{i=1}^{m}\left[e_{i}\left(v^{\prime}(t)\right)\left\langle\operatorname{grad} \rho_{N}, e_{i}\right\rangle+v^{\prime}(t) \operatorname{Hess}_{N} \rho_{N}\left(e_{i}, e_{i}\right)\right] \\
& =v^{\prime \prime}(t) \sum_{i=1}^{m} a_{i}^{2}+v^{\prime}(t) \sum_{i=1}^{m} \sum_{j=1}^{n-1}\left(c_{i}^{j}\right)^{2} \operatorname{Hess} \rho_{N}\left(\partial / \partial \theta_{j}, \partial / \partial \theta_{j}\right) .
\end{aligned}
$$

Since $v^{\prime}(t) \leq 0$ we have by the Hessian Comparison Theorem that

$$
\begin{aligned}
-\triangle f(x) \geq & -v^{\prime \prime}(t) \sum_{i=1}^{m} a_{i}^{2}-v^{\prime}(t) \frac{C_{\kappa}}{S_{\kappa}}(t) \sum_{i=1}^{m} \sum_{j=1}^{n-1}\left(c_{i}^{j}\right)^{2} \\
= & -v^{\prime \prime}(t) \sum_{i=1}^{m} a_{i}^{2}-v^{\prime}(t) \frac{C_{\kappa}}{S_{\kappa}}(t)\left[m-\sum_{i=1}^{m} a_{i}^{2}-\sum_{i=1}^{m} b_{i}^{2}\right] \\
= & -v^{\prime \prime}(t)-(m-2) v^{\prime}(t) \frac{C_{\kappa}}{S_{\kappa}}(t) \\
& +v^{\prime \prime}(t)\left[1-\sum_{i=1}^{m} a_{i}^{2}\right]-v^{\prime}(t) \frac{C_{\kappa}}{S_{\kappa}}(t)\left[1-\sum_{i=1}^{m} a_{i}^{2}+1-\sum_{i=1}^{m} b_{i}^{2}\right] \\
= & \lambda_{1}\left(B_{\mathbb{N} m-1}(\kappa)(r)\right) v(t) \\
& +v^{\prime \prime}(t)\left[1-\sum_{i=1}^{m} a_{i}^{2}\right]-v^{\prime}(t) \frac{C_{\kappa}}{S_{\kappa}}(t)\left[1-\sum_{i=1}^{m} a_{i}^{2}+1-\sum_{i=1}^{m} b_{i}^{2}\right] .
\end{aligned}
$$

We will show that the last line of (3.4) is nonnegative; this is

$$
v^{\prime \prime}(t)\left[1-\sum_{i=1}^{m} a_{i}^{2}\right]-v^{\prime}(t) \frac{C_{\kappa}}{S_{\kappa}}(t)\left[1-\sum_{i=1}^{m} a_{i}^{2}+1-\sum_{i=1}^{m} b_{i}^{2}\right] \geq 0 .
$$

Substituting $v^{\prime \prime}(t)=-(m-2) v^{\prime}(t) \frac{C_{\kappa}}{S_{\kappa}}(t)-\lambda_{1}\left(B_{\mathbb{N}^{m-1}(\kappa)}(r)\right) v(t)$ in (3.5) we obtain

$$
\begin{aligned}
& v^{\prime \prime}(t)\left[1-\sum_{i=1}^{m} a_{i}^{2}\right]-v^{\prime}(t) \frac{C_{\kappa}}{S_{\kappa}}(t)\left[1-\sum_{i=1}^{m} a_{i}^{2}+1-\sum_{i=1}^{m} b_{i}^{2}\right] \\
= & -\left[(m-1) v^{\prime}(t) \frac{C_{\kappa}}{S_{\kappa}}(t)+\lambda_{1}\left(B_{\mathbb{N}^{m-1}(\kappa)}(r)\right) v(t)\right]\left[1-\sum_{i=1}^{m} a_{i}^{2}\right] \\
- & v^{\prime}(t) \frac{C_{\kappa}}{S_{\kappa}}(t)\left[1-\sum_{i=1}^{m} b_{i}^{2}\right] \geq 0
\end{aligned}
$$


since we have that $(m-1) v^{\prime}(t) \frac{C_{\kappa}}{S_{\kappa}}(t)+\lambda_{1}\left(B_{\mathbb{N}^{m-1}(\kappa)}(r)\right) v(t)<0$ by Lemma (2.4) and $\left[1-\sum_{i=1}^{m} a_{i}^{2}\right] \geq 0$ and $\left[1-\sum_{i=1}^{m} b_{i}^{2}\right] \geq 0$. From (3.4) we have $-\frac{\triangle f}{f}(x) \geq$ $\lambda_{1}\left(B_{\mathbb{N}^{m-1}(\kappa)}(r)\right)$. Therefore,

$$
\lambda^{*}(\Omega) \geq \inf _{\Omega}(-\triangle f / f) \geq \lambda_{1}\left(B_{\mathbb{N}^{m-1}(\kappa)}(r)\right) .
$$

To prove the last assertion of Theorem 1.1 we need the following proposition proved in [5].

Proposition 3.1. Let $\Omega$ be a bounded domain in a smooth Riemannian manifold. Let $v \in C^{2}(\Omega) \cap C^{0}(\bar{\Omega}), v>0$ in $\Omega$ and $v \mid \partial \Omega=0$. Then

$$
\lambda^{*}(\Omega) \geq \inf _{\Omega}\left(-\frac{\triangle v}{v}\right) .
$$

Moreover, $\lambda^{*}(\Omega)=\inf _{\Omega}\left(-\frac{\triangle v}{v}\right)$ if and only if $v=u$, where $u$ is a positive eigenfunction of $\Omega$, i.e. $\triangle u+\lambda^{*}(\Omega) u=0$.

If we have equality $\lambda_{1}(\Omega)=\lambda_{1}\left(B_{\mathbb{N}^{m-1}(\kappa)}(r)\right)$, we have that $f$ is an eigenfunction and the expression (3.6) is zero (at each point of $\Omega$ ). This happens if and only if

$$
1=\sum_{i=1}^{m} \alpha_{i}^{2}=\sum_{i=1}^{m} \beta_{i}^{2}
$$

On the other hand, we can write at each point $x \in \Omega$

$$
\operatorname{grad}_{N}=\sum_{i=1}^{m} \alpha_{i} e_{i}+\left(\operatorname{grad}_{N}\right)^{\perp}
$$

where $\left(\operatorname{grad}_{N}\right)^{\perp}$ is normal to the tangent space of $T_{x} \Omega$. Likewise we can write

$$
\partial / \partial s=\sum_{i=1}^{m} \beta_{i} e_{i}+(\partial / \partial s)^{\perp} .
$$

Since $\left\|\operatorname{grad}_{N}\right\|^{2}=\sum_{i=1}^{m} \alpha_{i}^{2}+\left\|\left(\operatorname{grad}_{N}\right)^{\perp}\right\|$ and $\|\partial / \partial s\|^{2}=\sum_{i=1}^{m} \beta_{i}^{2}+\left\|(\partial / \partial s)^{\perp}\right\|^{2}$, we conclude that $\left(\operatorname{grad}_{N}\right)^{\perp}=0=(\partial / \partial s)^{\perp}$. Thus the tangent space $T_{x} \Omega$ contains the vectors grad $\rho_{N}$ and $\partial / \partial s$ for each $x \in \Omega$. Thus, we could have chosen in (3.3) an orthonormal basis for $T_{x} \Omega$ in the following way: $e_{1}=\operatorname{grad} \rho_{N}, e_{2}=\partial / \partial s$ and $\left\{e_{3}, \ldots, e_{m}\right\} \subset\left\{\partial / \partial \theta_{1}, \ldots, \partial / \partial \theta_{n-1}\right\}$. Clearly the set of vectors $\operatorname{grad}_{N}$ and $\partial / \partial s$ form smooth vector fields on $\Omega$ since they are the restrictions of smooth vector fields on $N \times \mathbb{R}$ to a smooth immersed submanifold. The integral curves of the vector field $\partial / \partial s$ in $\Omega$ are $\{x\} \times \mathbb{R}$ contained in $\varphi(M)$, and $\Omega=\varphi^{-1}\left(B_{N}(r) \times \mathbb{R}\right)$ is not bounded. This proves Theorem 1.1 .

3.2. Proof of Theorem 1.6. Let $\varphi: M \hookrightarrow N \times \mathbb{R}$ be a complete immersed $m$-submanifold with locally bounded mean curvature in $N \times \mathbb{R}$, where $N$ has radial sectional curvature bounded above $K_{N} \leq \kappa$ along the geodesics issuing from $x_{0}$. Define $\tilde{\rho_{N}}: N \times \mathbb{R} \rightarrow \mathbb{R}$ by $\tilde{\rho_{N}}(x, t)=\rho_{N}(x), \rho_{N}(x)=\operatorname{dist}_{N}\left(x_{0}, x\right)$. Let $\Omega(r)=\varphi^{-1}\left(B_{N}\left(x_{0}, r\right) \times \mathbb{R}\right), f=\tilde{\rho_{N}} \circ \varphi$ and $X=\operatorname{grad} f$. The idea is to choose 
$r<\min \left\{\operatorname{inj}_{N}\left(x_{0}\right), \pi / 2 \sqrt{\kappa}\right\}, \pi / 2 \sqrt{\kappa}=\infty$ if $\kappa \leq 0$, properly such that $\inf _{\Omega(r)} \operatorname{div} X>$ 0 . Then by Theorem 2.3 we have that

$$
\lambda^{*}(\Omega(r)) \geq\left(\frac{\inf \operatorname{div} X}{2 \sup |X|}\right)^{2} .
$$

Observe that $\operatorname{div} X=\triangle_{M} f$, and as in (2.2) we have

$$
\triangle_{M} f(x)=\left[\sum_{i=1}^{m} \operatorname{Hess}_{N \times \mathbb{R}} \tilde{\rho_{N}}\left(e_{i}, e_{i}\right)+\left\langle\operatorname{grad}_{N \times \mathbb{R}} \tilde{\rho_{N}}, \vec{H}\right\rangle\right](\varphi(x)),
$$

where $\vec{H}=\sum_{i=1}^{m} \alpha\left(e_{i}, e_{i}\right)$ is the mean curvature vector of $\varphi(M)$ at $\varphi(x)$ and $\left\{e_{1}, \ldots, e_{m}\right\}$ is an orthonormal basis of $T_{x} M$ as in (3.3) identified with $\left\{d \varphi \cdot e_{1}\right.$, $\left.\ldots, d \varphi \cdot e_{m}\right\}$. Now

$$
\begin{aligned}
\sum_{i=1}^{m} \operatorname{Hess}_{N \times \mathbb{R}} \tilde{\rho_{N}}\left(e_{i}, e_{i}\right) & =\sum_{i=1}^{m} \operatorname{Hess}_{N} \rho_{N}\left(e_{i}, e_{i}\right) \\
& =\sum_{i=1}^{m} \sum_{j=1}^{n-1}\left(c_{j}^{i}\right)^{2} \operatorname{Hess}_{N} \rho_{N}\left(\partial / \partial \theta_{j}, \partial / \partial \theta_{j}\right) \\
& \geq \sum_{i=1}^{m}\left(1-a_{i}^{2}-b_{i}^{2}\right) \frac{C_{\kappa}}{S_{\kappa}}(r)
\end{aligned}
$$

On the other hand $\left\langle\operatorname{grad}_{N \times \mathbb{R}} \tilde{\rho_{N}}, \vec{H}\right\rangle=\left\langle\operatorname{grad}_{N} \rho_{N}, \vec{H}\right\rangle$ and

$$
\begin{aligned}
\left\langle\operatorname{grad}_{N} \rho_{N}, \vec{H}\right\rangle & =\left\langle\left(\operatorname{grad}_{N} \rho_{N}\right)^{\perp}, \vec{H}\right\rangle \\
& \leq|H| \sqrt{1-\sum_{i=1}^{m} a_{i}^{2}} \\
& \leq h\left(x_{0}, r\right) \sqrt{1-\sum_{i=1}^{m} a_{i}^{2}}
\end{aligned}
$$

since $\left.\mid \operatorname{grad}_{N} \rho_{N}\right)\left.^{\perp}\right|^{2}=\left(1-\sum_{i=1}^{m} a_{i}^{2}\right)$. Therefore from (3.8) and (3.9) we have

$$
\triangle_{M} f(x) \geq(m-2) \frac{C_{\kappa}}{S_{\kappa}}(r)-h\left(x_{0}, r\right)>0 .
$$

We have two cases to consider. First is the case that $\left|h\left(x_{0}, r\right)\right|<\Lambda^{2}<\infty$ and we choose $r \leq \min \left\{\operatorname{inj}_{N}\left(x_{0}\right), \pi / 2 \sqrt{\kappa},\left(C_{\kappa} / S_{\kappa}\right)^{-1}\left(\Lambda^{2} /(m-2)\right)\right\}$. In case that $\lim _{r \rightarrow \infty} h\left(x_{0}, r\right)=\infty$ there is $r_{0}$ so that $(m-2) \frac{C_{\kappa}}{S_{\kappa}}\left(r_{0}\right)-h\left(x_{0}, r_{0}\right)=0$, since we can assume without loss of generality that $h\left(x_{0}, r\right)$ is a continuous nondecreasing function in $r$. Then we choose

$$
r \leq \min \left\{\operatorname{inj}_{N}\left(x_{0}\right), \pi / 2 \sqrt{\kappa},\left(C_{\kappa} / S_{\kappa}\right)^{-1}\left(h\left(x_{0}, r_{0}\right) /(m-2)\right)\right\} .
$$

In both cases we have

$$
\lambda^{*}(\Omega(r)) \geq\left[\frac{(m-2) \frac{C_{\kappa}}{S_{\kappa}}(r)-h\left(x_{0}, r\right)}{2}\right]^{2} .
$$

To prove Corollary 1.7just see that $\Omega\left(R_{p_{1}(M)}\right)=M$ and $\lambda^{*}(M)=0$. 


\section{REFERENCES}

1. J. Barta, Sur la vibration fundamentale d'une membrane. C. R. Acad. Sci. 204 (1937), 472473.

2. Pierre H. Bérard, Spectral geometry: direct and inverse problems. Lect. Notes in Math. 1207, Springer-Verlag, 1986. MR861271 (88f:58146)

3. M. Berger, P. Gauduchon, and E. Mazet, Le spectre d'une variété riemannienne. Lect. Notes Math. 194, Springer-Verlag, Berlin-New York, 1971. MR0282313 (43:8025)

4. G. P. Bessa and J. F. Montenegro, Eigenvalue estimates for submanifolds with locally bounded mean curvature. Ann. Global Anal. and Geom. 24 (2003), 279-290. MR1996771(2004f:53068)

5. G. P. Bessa and J. Fabio Montenegro, An extension of Barta's theorem and geometric applications. Ann. Global Anal. Geom. 31 (2007), no. 4, 345-362. MR2325220 (2008e:53112)

6. G. P. Bessa and J. Fabio Montenegro, On compact $H$-hypersurfaces of $N \times \mathbb{R}$. Geom. Dedicata 127 (2007), 1-5. MR 2338510

7. A. Candel, Eigenvalue estimates for minimal surfaces in hyperbolic space. Trans. Amer. Math. Soc. 359 (2007), 3567-3575. MR2302506 (2007m:53076)

8. I. Chavel, Eigenvalues in Riemannian Geometry. Pure and Applied Mathematics, 115, Academic Press, Inc., 1984. MR768584 (86g:58140)

9. S. Y. Cheng, P. Li and S.-T. Yau, Heat equations on minimal submanifolds and their applications. Amer. J. Math. 106 (1984), 1033-1065. MR761578 (85m:58171)

10. L.-F. Cheung and P.-F. Leung, Eigenvalue estimates for submanifolds with bounded mean curvature in the hyperbolic space. Math. Z. 236 (2001), 525-530. MR1821303 (2002c:53094)

11. L. Jorge and D. Koutroufiotis, An estimate for the curvature of bounded submanifolds. Amer. J. Math. 103 (1981), 711-725. MR623135 (83d:53041b)

12. L. Jorge and F. Xavier, A complete minimal surface in $R^{3}$ between two parallel planes. Ann. of Math. (2) 112 (1980), no. 1, 203-206. MR584079 (82e:53087)

13. F. J. Lopez, F. Martín and S. Morales, Adding handles to Nadirashvili's surfaces. J. Diff. Geom. 60 (2002), 155-175. MR1924594 (2003f:53013)

14. F. J. Lopez, F. Martín and S. Morales, Complete nonorientable minimal surfaces in a ball of $\mathbb{R}^{3}$. Trans. Amer. Math. Soc. 358 (2006), 3807-3820. MR2219000(2007a:53014)

15. F. Martín and S. Morales, A complete bounded minimal cylinder in $\mathbb{R}^{3}$. Michigan Math. J. 47 (2000), no. 3, 499-514. MR1813541 (2001m:53015)

16. W. Meeks and H. Rosenberg, The theory of minimal surfaces in $M^{2} \times \mathbb{R}$. Comment. Math. Helv. 80 (2005), no. 4, 811-858. MR2182702 (2006h:53007)

17. W. Meeks and H. Rosenberg, Stable minimal surfaces in $M^{2} \times \mathbb{R}$. J. Differential Geom. 68 (2004), no. 3, 515-534. MR2144539 (2006b:53007)

18. N. Nadirashvili, Hadamard's and Calabi-Yau's conjectures on negatively curved and minimal surfaces. Invent. Math. 126 (1996), 457-465. MR1419004 (98d:53014)

19. R. Schoen and S.-T. Yau, Lectures on Differential Geometry. Conference Proceedings and Lecture Notes in Geometry and Topology, vol. 1, International Press, Cambridge, MA, 1994. MR.1333601 (97d:53001)

The Abdus Salam International Centre for Theoretical Physics, 34014 Trieste, Italy Current address: Department of Mathematics, Universidade Federal do Ceara-UFC, Campus do Pici, 60455-760 Fortaleza-CE, Brazil

E-mail address: bessa@mat.ufc.br

Department of Engineering, Universidade Federal do Ceara-UFC, Campus Cariri, Av. Castelo Branco, 150, 60030-200 Juazeiro do Norte-CE, Brazil

E-mail address: silvana_math@yahoo.com.br 